\title{
Isolation of condensed tannins in individual size from grape seeds and their impact on astringency perception
}

\author{
Wen $\mathrm{Ma}^{1,2,3}$, Pierre Waffo-Téguo ${ }^{1,2}$, Michäel Jourdes ${ }^{1,2}, \mathrm{Hua} \mathrm{Li}^{3}$, and Pierre-Louis Teissèdre ${ }^{1,2, a}$ \\ ${ }^{1}$ Univ. de Bordeaux, ISVV, EA 4577, Unité de recherche OENOLOGIE, 33882 Villenave d'Ornon, France \\ 2 INRA, ISVV, USC 1366 OENOLOGIE, 33882 Villenave d'Ornon, France \\ ${ }^{3}$ College of Enology, Northwest A \& F University, Shaanxi 712100, China
}

\begin{abstract}
Astringency perception, as an essential parameter for high-quality red wine, is principally elicited by condensed tannins in diversified chemical structures. The influence of DP size of condensed tannins on astringency perception remains unclear for decades. In the present study, the astringency intensity of purified and identified grape oligomeric tannins (DP ranged from 1 to 5) was firstly explored. A novel non-solid phase strategy was used to rapidly exclude the galloylated PAs from the non-galloylated PAs and fractionate the latter according to their DP size. Then, a series of PAs with individual DP size and galloylation were purified. Furthermore, purified compounds were identified by both normal phase HPLC-FLD and reverse phase UHPLC-ESI-Q-TOF. Finally, the contribution of the astringency perception of the individual purified tannins was examined with a salivary protein binding ability test. The results were observed by HPLC-FLD and quantified by changes in PA concentration remaining in the filtrate. In summary, a new approach without a solid stationary phase was developed to isolate PAs according to their DP size. And a positive relationship between the DP of PAs and salivary protein affinity was revealed.
\end{abstract}

\section{Introduction}

Condensed tannins are oligomers and polymers of flavan3-ols units belonging to the flavonoid class of polyphenols that are widely distributed throughout the plant kingdom and their derived products. Owing to their considerable contribution to nutritional functions [1] and sensory properties [2], condensed tannins have attracted much interest in recent decades. In grape seeds and skins, condensed tannins present as a heterogeneous mixture involving various flavan-3-ol subunits and diversified degrees of polymerization, which probably corresponds to distinctive bioactivities. Given the lack of efficient purification methodologies, few individual tannins have been purified and identified. Hence, our knowledge on grape and wine condensed tannin molecules has to be limited at the several known monomeric, dimeric and trimeric proanthocyanidins. Quantification of the large condensed tannins in grape and wine remains problematic and their bioactivities are barely understood [3].

Astringency, an essential parameter for high-quality red wine, is an oral sensation involving dryness and puckering. So far, it is generally thought that the perception of astringency in wine is primarily due to condensed tannins derived from grape, their mechanism being principally explained by non-covalent interactions between condensed tannins and salivary protein. Structures of condensed tannins determine both the intensity and quality of the wine astringency perception [4]. The aims of this study was to develop an efficient strategy to isolate tannins both in non-galloylated forms and at individual

a e-mail: pierre-louis.teissedre@u-bordeaux.fr
DP and to reveal the chemical affinity between tannin DP and salivary protein binding abilities, which implicates for wine astringency.

\section{Method}

\subsection{Grape seed procyanidins extraction}

Grape seeds were removed by hand from grapes, lyophilized for 2 days and stored at $-20^{\circ} \mathrm{C}$. The frozen seeds were finally ground in a ball grinder. An ASE 350 Accelerated Solvent Extraction System (Dionex Corporation. Sunnyvale. CA) was used as previously [5] to extract the tannins from the ground seeds. The obtained solid residue was redissolved in $30 \mathrm{~mL}$ of water and lyophilized.

The extract was solubilized in $250 \mathrm{~mL}$ of water/ethanol $(95: 5, \mathrm{v} / \mathrm{v})$ and extracted three times with chloroform $(\mathrm{v}=$ $250 \mathrm{~mL}$ ) to remove lipophilic material. Then the aqueous phase was extracted three times with ethyl acetate $(\mathrm{v}=$ $250 \mathrm{~mL}$ ) to obtain two distinctive fractions [6]. The organic fraction was concentrated and lyophilized to obtain a dry powder. A crude oligomeric PAs extract was obtained.

\subsection{Non-solid phase fraction}

The centrifugal partition chromatography (CPC) apparatus was an FCPC 1000 provided by Kromaton Technologies (Saintes-Gemmes-sur-Loire, France). PAs were separated by a two-phase system ethyl acetate-ethanol-water $(6: 1: 5$, $\mathrm{v} / \mathrm{v} / \mathrm{v})$. For each injection, $5 \mathrm{~g}$ of extract were dissolved in $10 \mathrm{~mL}$ of the upper and lower phases $(50 / 50, \mathrm{v} / \mathrm{v})$ of the system and $0.45 \mathrm{~mm}$ filtered. Experiments were carried out in ascending mode at $1000 \mathrm{rpm}$ with a flow 
rate of $15 \mathrm{~mL} / \mathrm{min}$ for $140 \mathrm{~min}$. The fraction collector was set to 1 tube/min. Every five CPC tubes, an aliquot $(200 \mu \mathrm{L})$ was taken, evaporated, dissolved in $1 \mathrm{~mL}$ of $\mathrm{H}_{2} \mathrm{O} / \mathrm{MeOH}(50: 50, \mathrm{v} / \mathrm{v})$ and analyzed by UHPLC-ESIQ-TOF. When grouping the tubes, samples presenting the similar HPLC profiles were pooled together, evaporated in vacuo, suspended in water and freeze-dried. Five determined fractions were obtained.

\subsection{Preparative High Performance Liquid Chromatography Purification}

Purification was performed on a Luna HILIC column $(21.2 \times 250 \mathrm{~mm}, 5 \mu \mathrm{m}$, Phenomenex) by a Varian LC machine. The mobile phase consisted of acidified acetonitrile (Eluent A) and acidified aqueous methanol (Eluent B. Methanol: water. 95:5. v/v), both containing $0.025 \%$ trifluoroacetic acid. The flow rate was $22 \mathrm{~mL} / \mathrm{min}$ and eluent B followed this gradient: $0 \mathrm{~min}, 7 \% ; 57 \mathrm{~min}$, $37.6 \%$; $60 \mathrm{~min}, 100 \%$; $67 \mathrm{~min}, 100 \%$; $73 \mathrm{~min}, 7 \% ; 83$ min, $7 \%, 52 \mathrm{~min}$. For each injection, $100 \mathrm{mg}$ of fraction compounds were dissolved in $0.5 \mathrm{~mL}$ methanol and manually injected into the system. UV detection was carried out at $254 \mathrm{~nm}$ and $280 \mathrm{~nm}$.

\subsection{HPLC-FLD in normal phase analysis}

A Thermo-Finnigan Surveyor system was used for the normal phase HPLC analysis. This HPLC-UV system was also coupled to a Thermo-Finnigan LCQ Advantage spectrometer equipped with an electrospray ionization source and an ion trap mass analyzer. Fluorescence and mass data were analyzed by ChromQuest 4.2 and Xcalibur 2.2.0 software, respectively. Separation was performed on normal phase Luna HILIC column $(4.6 \times$ $250 \mathrm{~mm}, 5 \mu \mathrm{m}$, Phenomenex). The separation condition was reported previously [7]. Each sample was injected three times. Unknown concentrations were determined from the regression equations.

\subsection{Saliva binding ability test}

A pool of saliva was collected from 20 volunteers (10 males and 10 females aged 20 to 35 years old). They were in good health and not undergoing oral treatment. They were previously instructed to avoid smoking on the saliva donation day and take no food or beverages for atleast $1 \mathrm{~h}$ before collection. Collection time was standardized between 10-12 a.m. to reduce the concentration variability. Saliva was collected with no oral stimulus but rather with a visual stimulus by lemons. The saliva was collected by small bottles and immediately frozen at $-20^{\circ} \mathrm{C}$ after collection. After all the samples had been collected, they were thawed, pooled and refrozenfor lyophilization to concentrate the nature saliva around three-fold. After lyophilized concentration, the thawed saliva sample was centrifuged at $8,000 \mathrm{~g}$ for $5 \mathrm{~min}$ at $4^{\circ} \mathrm{C}$ by a Jouan MR22 refrigerated centrifuge. The supernatants were the targeted saliva protein sample.

The method was that of Schwarze and Hofman [8] with some modifications. Purified tannins (monomers, dimers, trimers, tetramers and pentamers) were prepared as a dissolution at the concentration of $1.5 \mathrm{mg} / \mathrm{mL}$ in model wine solution (ethanol $12 \%$; tartaric acid $=1 \mathrm{~g} / \mathrm{L} ; \mathrm{pH}=$ 3.5). Tannin solution $(300 \mu \mathrm{L})$ was mixed with $700 \mu \mathrm{L}$ of
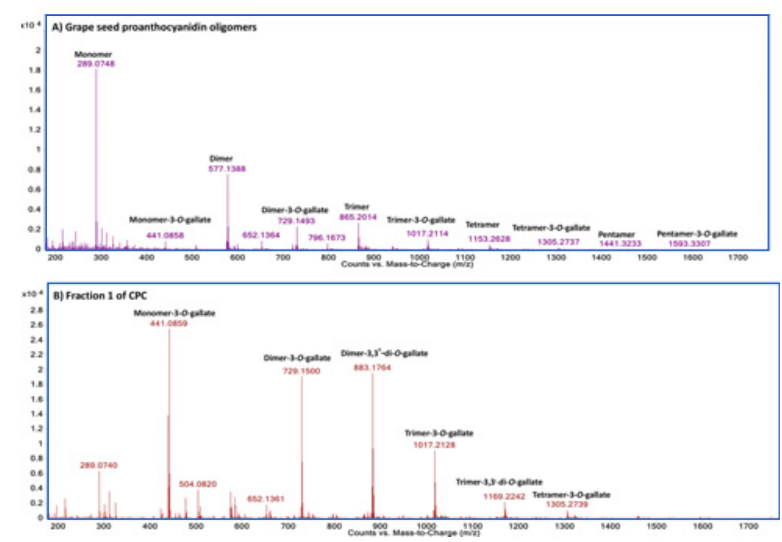

Figure 1. MS spectra obtained for crude extract (A) and fraction 1 of CPC (B).

prepared saliva sample or water (as control) and incubated at $37^{\circ} \mathrm{C}$ for $5 \mathrm{~min}$. After incubation, an aliquot $(400 \mu \mathrm{L})$ of the mixture was moved to a $3 \mathrm{k}$ Da centrifugal filter (Amicon Ultra-0.5 Centrifugal Filter 3k Devices, Merck Millipore) and centrifuged at $18,000 \mathrm{rpm}$ for $5 \mathrm{~min}$ at $37^{\circ} \mathrm{C}$. The filtrate in the bottom, namely the non-bound tannins, was injected into the normal phase HPLC-FLD for quantitative analysis. Each analysis was performed in duplicate.

\section{Results and discussions}

\subsection{Isolation of grape oligomeric tannins in individual DP size}

In the crude grape seed PA oligomer extract, PAs and their galloylated derivatives were found as a mix (Fig. 1A.). After CPC had run in ascending mode for 140 mins, six fractions were produced. The first $(\mathrm{F} 1,1.72 \mathrm{~g}, 40 \%)$ was comprised of multiple galloylated PAs (as shown in Fig. 1B). The main products of fraction two (F2, $1.04 \mathrm{~g}$, $21.8 \%)$, fraction three $(\mathrm{F} 3,546.9 \mathrm{mg}, 11.41 \%)$, fraction four (F4, 302.3 mg, 6.31\%) and fraction five (F5, 257.2mg, $5.36 \%$ ) corresponded to monomers, dimers, trimers and tetramers/pentamers, respectively. The application of CPC on grape PAs fractionation was less-time consuming, gave a high recovery, could potentially be scaled-up and was less expensive thanks to low solvent costs and the absence of expensive adsorbents. In general, the total recovery yield of CPC was $87.74 \%$, which was much higher than any traditional solid-phase separation strategies to date. Specifically, the untargeted compounds (galloylated PAs) with a high percentage were excluded in the very beginning, while the targeted PAs compounds were fractionated consecutively according to their DP. The mass response of dimers remained high in F4 and F5 owing to the huge quantity of dimers in the crude extract and the weak mass signals of the large PA molecules. Tetramers and pentamers were already present in the crude extract and were enriched in F5 after CPC. Thanks to the enrichment of tetramers and pentamers, the fifth fraction was well-prepared to be purified by a solid-phase chromatography with a low and efficient injection amount in the next step. PAs were purified from $4.8 \mathrm{~g}$ of oligomeric PA grape seed extract by the combination of CPC using a ternary biphasic system 

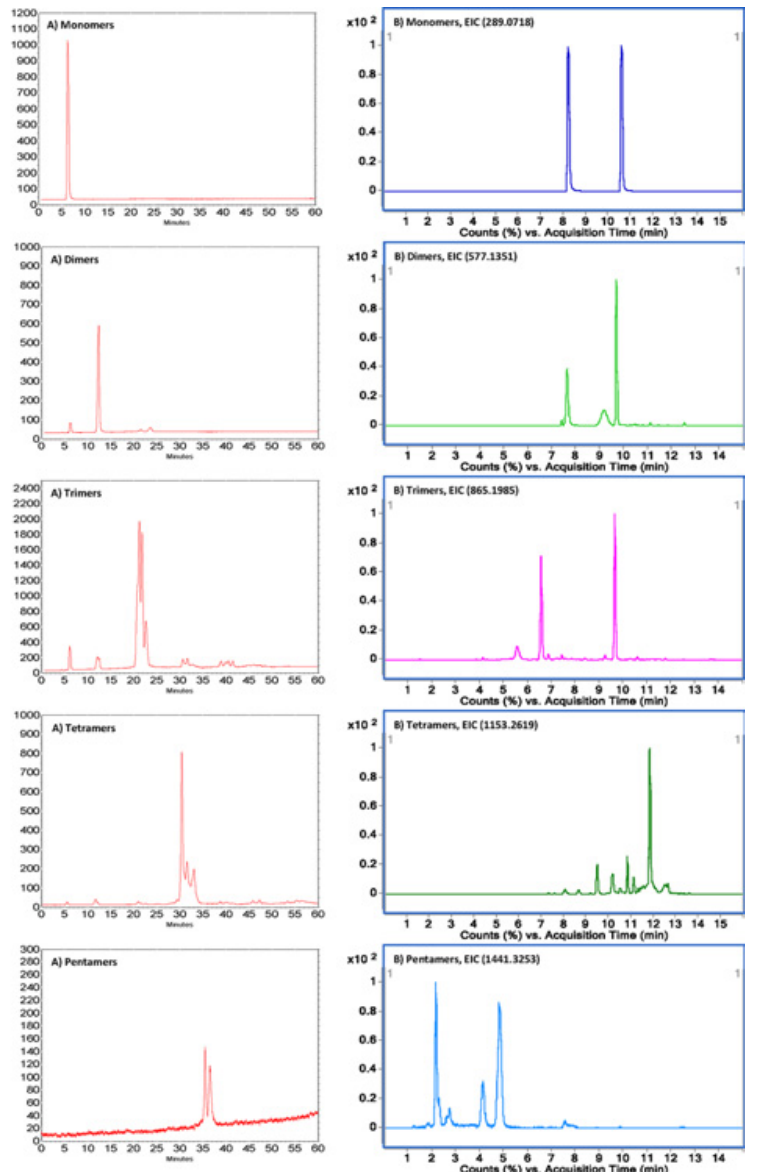

Figure 2. Two complementary and orthogonal HPLC approaches to identify the five purified PAs: A). Normal phase HPLCFLD chromatograms to separate PAs according to their DP; B). Reverse phase UHPLC-Q-TOF extracted ion chromatograms to separate isomeric PAs at the same DP.

EtOAc/EtOH/ $\mathrm{H}_{2} \mathrm{O}(6: 1: 5, \mathrm{v} / \mathrm{v} / \mathrm{v})$ and preparative normal phase HPLC. Although weak signals of hexamers and higher molecules could be detected by HRMS in the tail fraction, their quantities were too low to be isolated or to be used for salivary protein investigation. Individual oligomeric PAs were purified by preparative normal phase HPLC on a Luna HILIC column according to the HPLC gradient of Kelm [7] with the methods transformation. The PAs present as a heterogeneous mixture in grapes, which involves various isomers and are hardly available as pure compounds but rather as a mix [9]. Hence, the attempts to isolate each individual pure PAs molecules with high DP were time-consuming and not really necessary. Therefore, the normal phase column was used to isolate and identify oligomeric PAs according to DP in preparative HPLCUV and analytical HPLC-FLD, respectively. To our knowledge, this is the first time that PAs from grape have been purified in individual.

\subsection{Compounds identification by both HPLC-FLD and UHPLC-HRMS}

Identification of the purified compounds is illustrated in Fig. 2. by two complementary and orthogonal approaches: the normal phase HPLC-FLD system and a reverse phase UHPLC-QTOF system. A series of PAs with individual
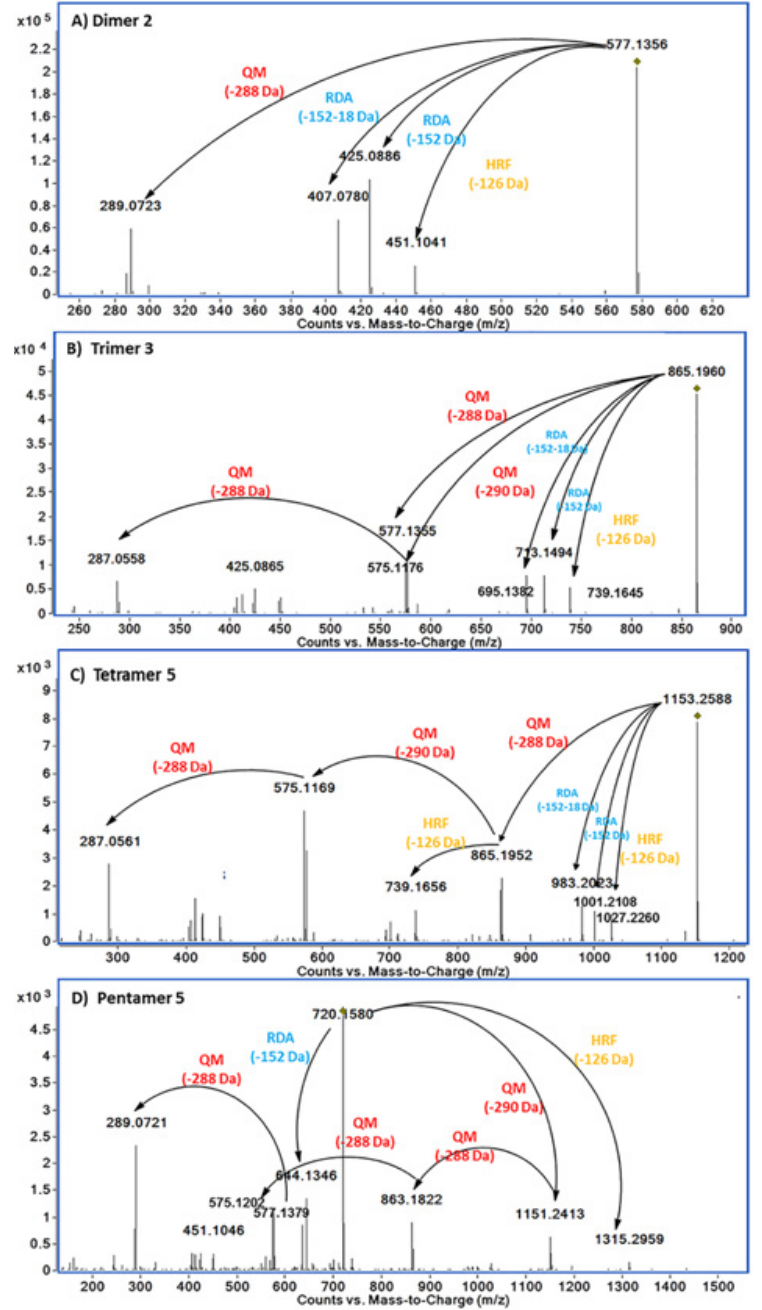

Figure 3. Examples of MS/MS fragments of purified dimeric, trimeric, tetrameric and pentameric PA.

DP were obtained: monomers (white powder; purity: 99.9\%), dimers (white powder; purity: $87.7 \%$ ), trimers (light yellow powder; purity: 83.2\%), tetramers (light yellow powder; purity: $92.5 \%$ ) and pentamers (light yellow powder; purity: 99.9\%). The purity was examined by HPLC-FLD (Fig. 2A) and the fluorescence absorbance declined with the rise in DP [28]. As shown in Fig. 2B, MS identification of compounds was performed with UHPLC-HRMS equipment in reverse phase. Two isomeric monomers, four isomeric dimers, three isomeric trimers, five isomeric tetramers and five isomeric pentamers were found and identified. As demonstrated in Fig. 3, the main fragmentation pathways of purified PAs in negative ion mode ESI-MSMS spectra were postulated based on the principles of quinone methide fission $(\mathrm{QM})$ with the ion lost from upper unit $(-288 \mathrm{Da})$ and ion lost from lower unit $(-290 \mathrm{Da})$, retro-Diels-Alder fission (RDA, $-152 \mathrm{Da}$ ) and a loss of water molecule $(-18 \mathrm{Da})[29,30]$. Dimer $2\left([\mathrm{M}-\mathrm{H}]^{-}, m / z\right.$ 577.1356, Fig. 3A) was diagnosed by the fragment ions with $\mathrm{m} / \mathrm{z}$ 451.1041, 425.0886, 407.0780, 289.0723. The ion with $\mathrm{m} / z 289.0723\left([\mathrm{M}-\mathrm{H}-\mathrm{QM}(288 \mathrm{Da})]^{-}\right)$was likely produced after QM cleavage of the $[\mathrm{M}-\mathrm{H}]^{-}$ion with the loss of upper unit (epi)catechin. The ions with $\mathrm{m} / \mathrm{z}$ 451.1041, 425.0886 , 407.0780 were corresponding to the fissions 


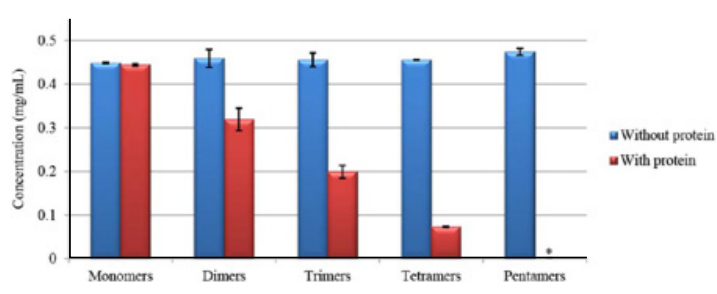

Figure 4. Examples of MS/MS fragments of purified dimeric, trimeric, tetrameric and pentameric PA.

of HRF (-126 Da), RDA (-152 Da) and RDA $+\mathrm{H}_{2} \mathrm{O}$ (-152-18 Da), respectively. Similarly, after QM cleavage, trimer 3 ([M-H] $]^{-}, m / z$ 865.1960, Fig. 3B) was fragmented into 577.1355 ([M-H-QM(288 Da)]-) and 575.1176 ([M$\left.\mathrm{H}-\mathrm{QM}(289 \mathrm{Da})]^{-}\right)$, with the loss of upper and lower unit, respectively. The fragments with $m / z 287.0558$ could be diagnosed by second fragmentation of either one upper unit loss from the $\mathrm{m} / \mathrm{z} 575.1196$ or one lower unit loss from the $m / z$ 577.1355. The ions with $m / z$ 739.1645, 713.1494, 695.1382 were corresponding to the fissions of HRF $(-126 \mathrm{Da}), \mathrm{RDA}(-152 \mathrm{Da})$ and $\mathrm{RDA}+\mathrm{H} 2 \mathrm{O}$ $(-152-18 \mathrm{Da})$ from the precursor ions $(\mathrm{m} / \mathrm{z} 865.1960)$, respectively.

In Fig. 3C, tetramer 5 was identified by the precursor ions $\left([\mathrm{M}-\mathrm{H}]^{-}, m / z\right.$ 1153.2588) and its fragment ions. Sequencing of this tetramer by QM fissions was straightforward, diagnosed by the fragment ions with $m / z 865.1952,575.1169,287.0561$. The ion with $\mathrm{m} / \mathrm{z}$ 1027.2260 and 739.1656 could be formed via a HRF fission from the precursor ion $(m / z$ 1153.2588) and one upper unit cleavage ion $(m / z 865.1952)$, respectively. The $m / z 1001.2108$ ion can result from an RDA of ring C of the precursor ions. The ion with $\mathrm{m} / \mathrm{z} 983.2023$ was derived from both RDA fission and loss of the equivalent of water $(18 \mathrm{Da})$. In Fig. 3D, the precursor ion ([M$2 \mathrm{H}]^{2-}, m / z$ of 720.1580$)$ was cleaved by QM fission into the ions with $m / z 1151.2413,863.1822,575.1202$, 289.0721 with the loss of the first, the second, the third and the forth units, respectively. The ion $m / z 644.1346$ was identified as a $[\mathrm{M}-2 \mathrm{H}]^{2-}$ ion after an RDA fission while the ion $m / z \quad 1315.2959$ was observed as a $[\mathrm{M}-\mathrm{H}]^{-}$ ion after HRF fission from the precursor ion. Hence, this compounds were diagnosed as (epi)catechin-(epi)catechin(epi)catechin-(epi)catechin-(epi)catechin.

\subsection{Relationship between tannin size and salivary protein binding abilities}

The harmony of high-quality red wine is mainly due to the balance of multiple flavors attributed to the numerous chemical components it contains [10]. Tannins are generally believed to interpret the axis of astringency perception. In this investigation, the astringency intensities of the purified PAs were examined by their ability to bind salivary protein and were quantified by HPLC-FLD [8]. A detector of FLD rather than UV was used in order to avoid the UV response of salivary protein at sizes below $3 \mathrm{k} \mathrm{Da}$. The amount of interacting tannins was calculated as the difference in tannin concentration in filtrate solution with and without salivary protein. As demonstrated in Fig. 4, an obvious decline in interacting tannin concentration from "with" to "without" saliva protein was observed for all of the five PAs. The monomers descended a little whereas there as a remarkable decrease $(0.14 \mathrm{mg} / \mathrm{mL})$ in the dimers. More than half of the trimers $(0.25 \mathrm{mg} / \mathrm{mL})$ were bound to salivary protein and even more tetramers $(0.38 \mathrm{mg} / \mathrm{mL})$ were bound. On the other hand, no pentamers were detected in the filtrate of the sample with salivary protein, indicating that the latter aggregated all the pentamers used in the test. This indicates a positive relationship between DP (ranged from one to five) and the salivary protein affinity of tannins. This result was in agreement with both the NMR interpretation of saliva protein binding ability to 4 procyanidin dimers (B14) and one trimer (C2) [11] and the previous astringency sensory studies on flavan-3-ols monomers, dimers and trimers chemically synthesized [12]. Unfortunately, we could not verify the inflexion point of DP, which was supposed previously [13], the isolation and identification of the grape tannins with higher DP are in need.

\section{Conclusion}

A simple rapid non-solid phase strategy has been developed to efficiently isolate PAs according to DP and to exclude the galloylated PAs. Monomeric, dimeric, trimeric, tetrameric and pentameric PAs were first purified from grape, thereby providing more substances for PA quantification in grape/wine and for further investigations concerning their bioactivity. Furthermore, a tentative test on salivary protein-binding capability was conducted to explore their astringency-stimulating abilities. This is the first report of the astringency activities of identified grape tannins without galloylated forms and in specific DP up to five. The findings of this investigation suggest that the capability of large tannin oligomers to be bound to salivary protein is much stronger than we estimated. Future research focusing on the bioactivities of large PAs molecular are now required.

\section{References}

[1] I. Ky, P. L. Teissedre, Molecules, 20, 2190-2207 (2015); I. Ky, B. Lorrain, N. Kolbas, A. Crozier, P. L. Teissedre, Molecules, 19, 482-506 (2014)

[2] B. Lorrain, S. Tempere, N. Iturmendi, V. Moine, G. de Revel, P.-L. Teissedre, Food Chemistry, 140, 76-82 (2013); K. Chira, M. Jourdes, P.-L. Teissedre, European Food Research and Technology, 234, 253-261 (2012)

[3] H. Li, J. Su, W. Ma, A. Guo, Z. Shan, H. Wang, FEMS yeast research, 15, fou010 (2015)

[4] W. Ma, A. Guo, Y. Zhang, H. Wang, Y. Liu, H. Li, Trends in Food Science and Technology, 40, 6-19 (2014)

[5] M. Reyes Gonzalez-Centeno, M. Jourdes, A. Femenia, S. Simal, C. Rossello, P.-L. Teissedre, Journal Of Agricultural And Food Chemistry, 60, 11850-11858 (2012)

[6] K. Chira, B. Lorrain, I. Ky, P. L. Teissedre, Molecules, 16, 1519-1532 (2011)

[7] M. A. Kelm, J. C. Johnson, R. J. Robbins, J. F. Hammerstone, H. H. Schmitz, Journal of agricultural and food chemistry, 54, 1571-1576 (2006) 
[8] B. Schwarz, T. Hofmann, European Food Research and Technology, 227, 1693-1698 (2008)

[9] A. L. Waterhouse, S. Ignelzi, J. R. Shirley, American Journal of Enology and Viticulture, 51, 383-389 (2000)

[10] É. Peynaud, J. Blouin, Le goût du vin, 5 ed., Dunod Paris, France, 2013
[11] O. Cala, N. Pinaud, C. Simon, E. Fouquet, M. Laguerre, E. J. Dufourc, I. Pianet, The FASEB Journal, 24, 4281-4290 (2010)

[12] H. Peleg, K. Gacon, P. Schlich, A. C. Noble, Journal of the Science of Food and Agriculture, 79, 1123-1128 (1999)

[13] A. G. Lea, in Plant polyphenols, 1992, pp. 827-847 